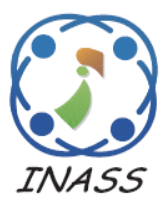

\title{
Modified Circular Fuzzy Segmentor and Local Circular Encoder to Iris Segmentation and Recognition
}

\author{
Emmanvel Raj Manohar Chirchi ${ }^{{ }^{*}}$ \\ Kharadkar Ravindra Digambar ${ }^{2}$ \\ ${ }^{1}$ Dr. Babasaheb Ambedkar Marathwada University, Aurangabad, India \\ ${ }^{2}$ Gyanchand Hirachand Raisoni Institute of Engineering \& Technology, Pune, India \\ *Corresponding author's Email: emmanvelraj1274@gmail.com
}

\begin{abstract}
Currently a lot of biometric procedures are being developed based on different features and algorithms. Nevertheless, it is known that, from all of these techniques, iris recognition is one of the most promising for high security applications. In this paper, a novel scheme is proposed to iris segmentation and recognition in iris based biometric system. In the new scheme, we use the modified circular fuzzy segmentor (MCFS) model to segment the pupil and iris inner boundary. After that, a binary encoder based feature extraction scheme named as LCE is proposed to extract the significant features to do the iris recognition process. Once feature extraction scheme is done by the LCE operator, the iris recognition is done through fuzzy logic classifier. We use three datasets from widely used iris databases (CASIA, MMU and UBIRIS) to analyze the increase of the error rates when the iris is inaccurately segmented. We selected 780 images of the CASIA, MMU and UBIRIS databases that the used segmentation algorithm can accurately segment. From the experimentation results, the proposed method of MCFS+LCE is outperformed than the existing methods.
\end{abstract}

Keywords: Modified circular fuzzy segmentor, Fuzzy logic classifier, Local circular operator, CASIA, MMU, UBIRIS.

\section{Introduction}

Today, security is one of the significant factors in the field of information, business, e-commerce, military and more [1]. For this motive, personal identification has turn out to be a considerable topic and some broadly employed methods of recognition such as PIN (Personal Identification Number), password, ID card and signatures have some disadvantages [2, 3-4]. ID card or PIN can be stolen, password may be forgotten and signatures can be reproduced. In the modern society, biometrics, the technology of carrying out personal identification or authentication through an individual's physical attributes, is turning into an increasingly feasible solution for identity management, information protection, and homeland security [5].

In common, biometrics engages the employ of physiological and behavioural features. The physiological features contain the subsequent: face, fingerprint, iris pattern, retina, ear, thermogram, hand geometry, palm-vein pattern, and the behavioural features comprise the subsequent: gait, keystroke dynamics, smell, signature, and voice [6, 7-9]. It is commonly granted that iris recognition is the most precise of all the biometric technologies applied for human authentication today. Above all, iris recognition is generally more user friendly and the physical subsistence of the user can be assured [10]. The human iris has been found to hold highly distinct features distinctive to each individual, and, thus, offers a good substitute resource for personal identification [11]. The iris is a distinctive organ that is composed of pigmented vessels and ligaments forming unique linear marks, slight ridges, grooves, furrows, vasculature, and other related features and marks. Comparing more features of the iris raises the likelihood of uniqueness. Therefore, for recognizing an individual, iris is declared to be the most accurate and dependable and has received widespread attentions over the last decade $[12,13-$ 16].

In this paper, our intention consists in the 
analysis of the relationship between the accuracy of the segmentation process and the error rates of typical recognition systems. In order to achieve this, we perform the following experimental procedure:

$>$ Selection of 780 images of the UBRIS (first version), CASIA and MMU databases. Manual verification that the used iris segmentation algorithm is able to accurately segment all the images.

$>$ A segmentation algorithm namely modified circular fuzzy segmentor (MCFS) is proposed to extract the region of interest (ROC) like pupil and iris identification.

$>$ Feature extraction on accurately segmented images: A binary texture feature called as local circular encoder (LCE) is proposed to extract significant iris features to do the recognition process.

$>$ Recognition: Recognition is done by the fuzzy logic classifier, which is divided the input iris image into whether recognize or not.

The outline of this paper is follows: a brief review of some of the literature works in iris recognition is presented in Section 2. Contribution of the paper is discussed in section 3. Section 4 is presented the dataset description. The proposed iris segmentation and recognition model is detailed in Section 5. The experimental results and performance evaluation discussion is provided in Section 6. Finally, the conclusions are summed up in Section 7.

\section{Review of Recent Works}

Using iris an abundant of researches has been suggested to attain personal identification and verification. A very few replicas utilize different iris segmentation algorithms for presenting more recognition among them. A robust iris recognition method has been offered by Y. Song et al [17] based on a sparse error correction model. In their method, all the training images are concatenated as a dictionary and the iris recognition task is cast to an optimization problem to look for a sparse representation of the test sample in terms of the dictionary. However, the overall complexities of their proposed algorithm become high. Moreover, an algorithm for iris recognition has been offered by $\mathrm{K}$. Miyazawa et al. [18] using phase-based image matching technique by means of phase components in 2D discrete Fourier transforms (DFTs) of specified images. But, it suffers to handle noisy conditions in illumination and camera to-face distances.

A method known as An Optimized Wavelength
Band Selection for Heavily Pigmented Iris Recognition has been brought in by Y. Gong et al [17]. Commercial iris recognition systems usually obtain images of the eye in 850-nm band of the electromagnetic spectrum. The greatly pigmented iris images are incarcerated at 12 wavelengths, from 420 to $940 \mathrm{~nm}$ in their work. Their experimental effects proposed that there exists a most appropriate wavelength band for heavily pigmented iris recognition when employing a single band of wavelength as illumination. A possibilistic fuzzy matching approach with invariant properties has been suggested by C. C. Tsai et al [19], which can offer a robust and efficient matching scheme for two sets of iris feature points. In addition, they suggested an efficient iris segmentation method to process the identified inner and outer boundaries to soft curves. Nevertheless, the complexities of their iris pupil identification become high

In addition, a perturbation-enhanced feature correlation filter (PFCF) has been suggested by $\mathrm{M}$. Zhang et al [20] for robust iris matching. In addition, accordingly as the gallery templates a set of further correlation filters are improved. The decision is found out by the fusion result of multiple correlation filters. In their system, the inner pupil boundary accurately for most samples from the database but fails to localize the outer iris boundary. In addition, a method for the recognition of iris patterns has been suggested by H. Rai et al [21] using the mixture of support vector machine and Hamming distance. Their approach as well employed parabola detection and trimmed median filter for the intention of eyelid and eyelash detection and removal, correspondingly. They showed that their method is computationally efficient and dependable. A weighted co-occurrence phase histogram (WCPH) has been offered by P. Lia et al [22] for symbolizing the local features of texture pattern and employs it to iris recognition. They assessed the performance of their technique on the UBIRIS.v2 database and assessed the vigor to noise of iris encoding and matching methods on the UBIRIS.v2 database. Nevertheless, their approach still suffers due to localization errors increase for the noisy for UBIRIS databases.

\section{Contribution of the Proposed Approach}

Two major contributions are made in the research in segmentation and recognition processes:

a) Segmentation: The design of a novel algorithm for segmentation is proposed called Modified Circular Fuzzy Iris Segmentor (MCFS) to identify the pupil and iris region. In addition, MCFS gives a 
complete and fulfilment segmentation structure to do easily recognition process.

b) Feature extraction: A binary encoder based feature extraction scheme namely as LCE is proposed to extract suitable features for iris recognition. The binary encoder based texture feature is suitable to do iris recognition, also, it can preserve the texture feature of the image by the neighbourhood pixel replacement process for every block.

\section{Eye Image Dataset}

Many eye image datasets can be publically accessed for iris recognition research [23, 24-25]. In the present study, the proposed method three datasets like CASIA, MMS and UBIRIS.

UBIRIS: The UBIRIS iris image databases consist of three subsets, namely UBIRIS v1.0 Session 1, Session 2 and UBIRIS v2.0 Train, with about 2377 images in total. Initially, under natural lighting and heterogeneous imaging conditions UBRIS database were incarcerated, which contains 10 photographs of each eye. The UBRIS database has two separate versions.

UBIRIS.v1 - In two separate sessions, this edition of the database contains 1877 images gathered from 241 eyes during September 2004. It reproduces less constrained imaging conditions. It is open and free obtainable.

UBIRIS.v2 - The second edition of the UBIRIS database has over 11000 images (and continuously growing) and more practical noise factors. Images were really incarcerated at-a-distance and on-themove.

MMU: MMU1 iris database contributes a total number of 450 iris images, which were taken using LG IrisAccess ${ }^{\circledR} 2200$. This camera is semiautomated and it operates at the range of $7-25 \mathrm{~cm}$. On the other hand, MMU2 iris database consists of 995 iris images. The iris images are collected using Panasonic BM-ET100US Authenticam and its operating range is even farther with a distance of 47 $53 \mathrm{~cm}$ away from the user. These iris images are contributed by 100 volunteers with different age and nationality. They come from Asia, Middle East, Africa and Europe. Each of them contributes five iris images for each eye. There are five left eye iris images which are excluded from the database due to cataract disease. Due to some privacy issue, anyone who interests on this database should email a particular name, institution and country to the administrators.

\section{An Intelligent Framework for Iris Recognition}

As summarized in figure 1, the main steps of our method are shown as follows:

* The system starts with the segmentation stage, followed by feature extraction and lastly, recognition. First, pupil and irises are segmented and segmentations are evaluated.

* Secondly, they are normalized by rubber sheet model to convert the rectangular conversion process. Then, a feature set is constructed based on LCE algorithm, which is newly designed operator for code generation process.

* After getting the texture features, at last, fuzzy logic classifier is used for accurately and inaccurately segmented irises separately for recognition.

\subsection{Segmentation through Modified Circular Fuzzy Segmentor (MCFS)}

Iris segmentation is pretty difficult because of all kinds of negative aspects of iris images, such as motion blurred, glasses, eyelids, eyelashes and reflection etc. Accurate segmentation is necessary for an accurate iris recognition system. In this section, we modified the recently developed technique named as circular fuzzy iris segmentation (CFIS), where N. P. Bodorin [26] has presented an approach to iris segmentation. In the classical iris segmentation procedures, papillary and limbic boundaries are identified by solving threedimensional optimization problems in order to and a radius and two center coordinates via gradient ascent or by using the Hough Transform or by iterating active contours. In our study, a modification is done on the vertical and horizontal run length quantization to extract the iris segmentation. Here, modified k-means quantization process is applied to improve the segmentation process. This part presents our segmentation scheme for iris and pupil regions.

The proposed method of modified circular fuzzy segmentation (MCFCS) is, 


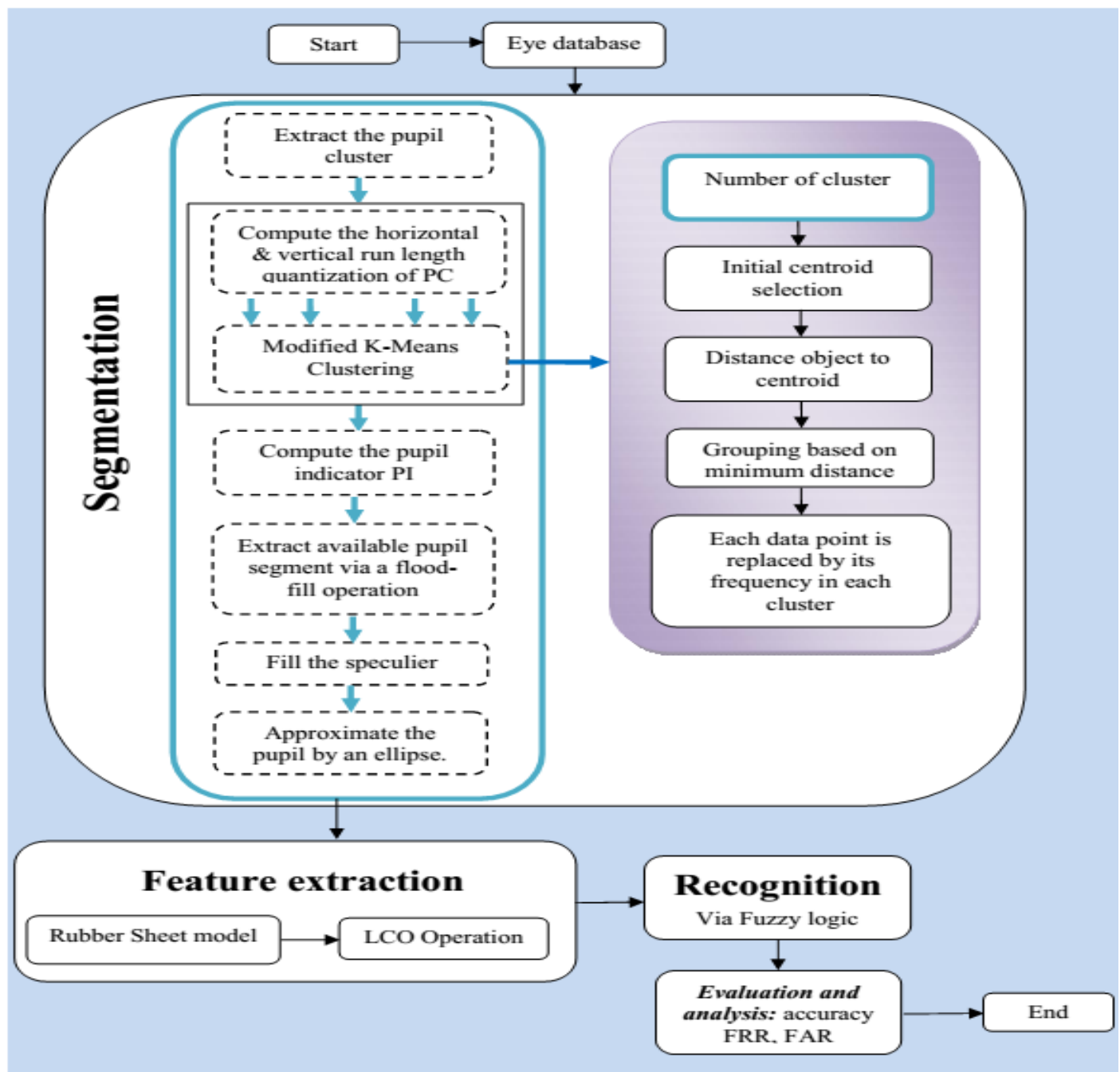

Figure.1 Framework of the proposed intelligent iris recognition system

\section{Step 1: Binarization}

At first, the input iris image is converted into binary image, which means it converts an image of up to 256 gray levels to a black and white image. The input image is given with a threshold value and it categorizes all pixels with values above this threshold as white, and all other pixels as black in image binarization process. Threshold is selected based on the image contrast (in this work, we use $\mathrm{T}=0.5$ since the gray image is in double format). Binarized Image,

$$
B_{\text {Binary }}(k, y)= \begin{cases}0, & \text { if } B_{\text {grey }}(k, y)=<\text { Threshold } \\ 1, & \text { Otherwise }\end{cases}
$$

\section{Step 2: Compute the horizontal \& vertical run length quantization}

- After converting to binary image, the run length encoding (RLE) is applied for code representation as a pair representation based on its histogram. For example, if $V=[1,1,1,1,0,0,1,1,1,1,1,1,1,1]$ is a vector to be encoded, then the run length encoding of $V$ is $H(V)=[(1,4),(0,2)(1,8)]$.

- Subsequently, run length quantization is applied here as a procedure to replace all ones within a binary image with the corresponding run length coefficients requantized in unsigned 8-bit integer domain by some custom quantization function as follows:

$\operatorname{rqf}(V(n))=\min (255, \max (1, \operatorname{round}(255 * V(n) / \max (V(n))))) \quad$ (2)

Where, $V$ is the vector to be quantized and $n$ is the index of its non-zero components.

Above process is done by two directions like vertical and horizontal and corresponding quantized image is obtained.

\section{Step 3: Modified Fast k-Means Image Quantization}


Thereafter, modified Fast k-Means Image Quantization (MFKMQ) is applied on the horizontal and vertical run length quantized image to obtain pupil region. Commonly, Fast k-Means Image Quantization (FKMQ) is a variant of k-means algorithm designed for fast chromatic clustering in uint8 domain. It transforms the input image in an equipotential chromatic map [20] with k-levels by replacing each chromatic value with the closest centroid. A suboptimal (incomplete) variant of FKMQ [25] can be easily derived by imposing termination in a small number of iterations while resetting the first centroid to the minimum available value (or to zero). In this way, the input image is forced to reveal its own range of darkness (numerical meaning of darkness according to the image histogram) and to return a handler to that area covered by lower chromatic values. Due to the modification in the FKMQ, the pupil region is accurately extracted, which improves the performance of the FKMQ. This is particularly useful in detecting the pupil location.

The procedure of applying the modified k-means clustering algorithm in our approach to the grey image consists of the following steps:

i. Determine the numbers of clusters $k_{1}, k_{2}$ and $k_{3}$ for three k-means clustering to form three clustering results of the same image $I$.

ii. For each single k-means clustering, randomly select $K_{i}, i=1,2, \ldots M$ pixels in grey color space as the initial clustering centroids. To calculate distance from center over particular attributes mathematical formula. (1-probability of instance value on that category) is used. The objective function is formulated as:

$$
J=\sum_{j=1}^{k} \sum_{i=1}^{n} d\left(X_{i}, Q_{i}\right)
$$

Where $d\left(X_{i}, Q_{i}\right)$ is the distance of data object $X_{k}$ from cluster center $Q_{i}$. This distance measure is formulated as

$$
d\left(X_{i}, Q_{j}\right)=\sum_{b=1}^{m} 1-q_{i b}, \quad \forall d, d=1,2,3, \ldots, m
$$

and cluster center is representative vector for cluster $i$ is defined as

$$
Q_{j}=\left[q_{i 1}, q_{i 2}, \ldots, q_{i m}\right]
$$

Where, $m$ is the number of attributes,

$$
q_{i m}=\frac{\left[F r e_{i m 1}, F r e_{i m 2}, \ldots, F r e_{i m d}\right]}{N}
$$

Where, Fre $e_{\text {imd }}$ is the frequency of value $d$ for the attribute $m$ in cluster $i$. iii. For each pixel in the image, assign pixel to the group that has the closest centroid based on the predetermined distance measure using equation (A).

iv. When all pixels have been assigned, recalculate the positions of the current clustering $K_{i}, i=1,2,3$ centroids.

\section{Step 4: Pupil identification}

Based on the vertical and horizontal pupil quantized image, the common region is selected and this region is named as pupil cluster.

\section{Step 5: Iris segmentation}

Finally, a circle is drawn named as iris region using the pupil circle.

\subsection{Feature extraction}

Once the iris segmentation is over, eyelid and eyelash pixels are identified using MCFS method and then the feature extraction is performed using Local Circular Encoder (LCE) algorithm. To start this method, Daugman's Rubber Sheet Model is used to convert circular to rectangular conversion (CTRC) operation. The main task of it is to generate a rectangular strip from segmented iris to form a rectangular matrix with a consistent value. Here, the iris area i.e. polar coordinate is converted Cartesian coordinates. Therefore, iris area is obtained as a normalized strip with regard to iris boundaries and pupillary centre. The homogenous rubber sheet model is devised by Daugman in 1993 used for normalization process. Then, the rectangular iris image is applied for the code generation process using LCE method.

\section{* Daugman's Rubber Sheet Model}

Once the iris image is efficiently localized, then the next step is to transform it into the rectangular sized fixed image. The transformation process is carried out using the Daugman's Rubber Sheet Model [25].

Using the following equation, the iris region is transformed to a 2D array with horizontal dimensions of angular resolution and vertical dimension of radial resolution.

$$
I[x(r, \theta), y(r, \theta)] \rightarrow I(r, \theta)
$$

Where, $I(x, y)$ is the iris region, $(x, y)$ and $(r, \theta)$ are the Cartesian and normalized polar coordinates respectively. The range of $\theta$ is $\left[\begin{array}{ll}0 & 2 \pi\end{array}\right]$ and $r$ is $\left[\begin{array}{ll}0 & 1\end{array}\right]$. $x(r, \theta)$ and $y(r, \theta)$ are defined as linear combinations set of pupil boundary points. The formulas given in 
the following equations perform the transformation,

$$
\begin{gathered}
x(r, \theta)=(1-r) x_{p}(\theta)+x_{i}(\theta) \\
y(r, \theta)=(1-r) y_{p}(\theta)+y_{i}(\theta) \\
x_{p}(\theta)=x_{p 0}(\theta)+r_{p} \operatorname{Cos}(\theta) \\
y_{p}(\theta)=y_{p 0}(\theta)+r_{p} \operatorname{Sin}(\theta) \\
x_{i}(\theta)=x_{i 0}(\theta)+r_{i} \operatorname{Cos}(\theta) \\
y_{i}(\theta)=y_{i 0}(\theta)+r_{i} \operatorname{Sin}(\theta)
\end{gathered}
$$

Where, $\left(x_{p}, y_{p}\right)$ and $\left(x_{i}, y_{i}\right)$ are the coordinates on the pupil and iris boundaries along the $\theta$ direction. $\left(x_{p 0,} y_{p 0}\right),\left(x_{i 0}, y_{i 0}\right)$ are the coordinates of pupil and iris centers [27].

\section{* Local circular encoder (LCE)}

Once the rectangular conversion is over, the code generation is done through the Local Circular Encoder (LCE). In order to design, weighted-based neighbour computation process is presented to remove or process that corrupted iris parts based on the quality assumption. The weighted-based neighbour computation is done by three domain information like spatial, Gabor and wavelet. The detailed process of the LCE method is presented in this subsection:

i.Let the spatial domain image (rectangular array) be denoted as $I(i, j)$, the wavelet domain image (via LL band) be denoted as $I_{w}(i, j)$ and the Gabor domain image (using Gabor filter $I_{w}(i, j)$ ) be denoted as $I_{g}(i, j)$, the resultant binary code through wavelet domain $I_{w}(i, j)$ be denoted as $\operatorname{code}_{A}$, the resultant binary code through Gabor domain $I_{g}(i, j)$ be denoted as $\operatorname{code}_{B}$, the resultant binary code through the original rectangular array $I(i, j)$ be denoted as code $e_{C}$, the obtained single binary 8-bit code via OR operation can be denoted as $B_{s}$.

ii.Partition the rectangular array $I(i, j)$ into $3 * 3 \mathrm{sub}$ blocks with centre block $X_{C}$.

iii.Apply a Gabor filter to the resultant rectangular array image $I(i, j)$ (original) to extract texture information. Therefore, the texture of iris is encoded by 2D multi-directional Gabor filter. Typically, an input array $I(i, j),(i, j) \epsilon \Omega(\Omega$ - set of image points), is convolved with a 2-D Gabor function $g(i, j),(i, j) \in \Omega$ to obtain a Gabor feature image $r(i, j)$ and the resultant binary code as follows:

$$
r(i, j)=\iint_{\Omega} I(\xi, \eta) g(i-\xi, j-\eta) d \xi d \eta
$$

Finally,

$$
\mathrm{Code}_{A} \leftarrow[r(i, j)]_{3 \times 3}
$$

iv.On other hand, a wavelet transform is applied to extract low and high band information. Following that, it decomposes four sub bands like LL, LH, HL and HH. In this study, LL band is used to computation process. According to this, binary code is generated from each $3 * 3$ block in LL sub band.

$$
\mathrm{Code}_{B} \leftarrow\left[I_{w}(i, j)\right]_{3 \times 3}
$$

In addition, the resultant binary code is obtained through the original rectangular array $I(i, j)$ (spatial domain). Mathematically, this transformation can be represented as

$$
\text { Code }_{C} \leftarrow[I(i, j)]_{3 \times 3}
$$

v.By combining these three domain information (i), (ii) and (iii) using OR operation, we obtain single 8-bit binary code, it can be represented as follows

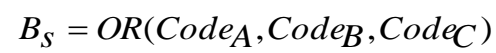

vi.The 8-bit binary code $B_{s}$ is converted as equal decimal number $D_{s}$ and it is replaced in same position of $I(i, j)$. This process is repeated for all $3 * 3$ blocks to obtain $I_{\text {out }}(i, j)$.

\subsection{Recognition via Fuzzy logic system}

In this study, a subtractive clustering technique (fuzzy logic) is used to recognize the two iris images. The genfis 2 algorithm provided by the MATLAB software uses a subtractive clustering method to generate a Fuzzy Interference System (FIS). Generally, Adaptive neuro fuzzy inference system (ANFIS) is a kind of neural network that is based on Takagi-Sugeno fuzzy inference system. Since it integrates both neural networks and fuzzy logic principles, it has potential to recognition process.

We have applied a fuzzy logic system using subtractive clustering for the recognition of two samples. To start the modelling process, an initial fuzzy model has to be derived. Here genfis2 is utilized to initialize Fuzzy Interference System (FIS) training by first applying the subtractive clustering [22] on the data. Using SUBCLUST function provided by MATLAB software to extract the set of rules that models the behaviour of the data, we can determine the number of rules and antecedent membership function. SUBCLUST function simply finds the optimal data point to define the cluster centers, based on the density of the surrounding data points. In addition, this model is required to find the number of inputs, number of linguistic variables and hence the number of rules in the fuzzy model. The initial model is also required to select the input variables for the final model and also the model selection criteria, before the final optimal model can be derived. The system generates one output from 
input, inputs and provided rules.

Membership function: In this model, the subtractive clustering is used to design membership function in input and output data points. Totally, two types of membership functions are used. In this study, Gaussian membership function is used for the input data and triangular membership function is used for output data.

Rule base: In addition, the fuzzy rules are generated using subtractive clustering. Here, rule should have two different decisions like as YES and NO. From the rule set, sample rules are presented as follows:

$$
\begin{gathered}
\boldsymbol{R}_{1}: I F\left(X_{1} \text { is } C_{1}\right) A N D\left(X_{2} \text { is } C_{1}\right) A N D\left(X_{3} \text { is } C_{2}\right) \\
A N D\left(X_{4} \text { is } C_{1}\right) A N D \ldots\left(X_{N} \text { is } C_{2}\right)
\end{gathered}
$$

\section{THEN $Y_{0}$ is 1}

$$
\begin{gathered}
\boldsymbol{R}_{2}: \operatorname{IF}\left(X_{1} \text { is } C_{1}\right) \quad O R \quad\left(X_{2} \text { is } C_{1}\right) \text { OR }\left(X_{3} \text { is } C_{2}\right) \quad \text { OR } \\
\left(X_{4} \text { is } C_{1}\right) \text { OR } \ldots\left(X_{N} \text { is } C_{2}\right)
\end{gathered}
$$

\section{THEN $Y_{0}$ is 0}

\section{Recognition}

The recognition of iris sample is carried out using the fuzzy system designed in the previous subsection. The testing iris image $N$ is given to the fuzzy logic system, where the test iris data is converted to the fuzzified value based on the fuzzy membership function. Then, the fuzzified input is matched with the fuzzy rules defined in the rule base. Here, the rule inference procedure is used to obtain the linguistic value that is then converted to the fuzzy score using the average weighted method. From the fuzzy score obtained, the decision is generated whether the test iris image belongs to the recognition or not.

\section{Simulation Results and Discussion}

This section presents the results obtained from the experimentation and its detailed discussion about the results. The proposed approach of iris segmentation and recognition model is experimented with the benchmark iris datasets and the result is evaluated with the sensitivity, specificity and accuracy. The proposed approach experiments has been carried out by a system with Intel (R) Core i5 processor, 3.20 GHz, 4 GB RAM and the operation system platform is Microsoft Windows 7 Professional. The programming language used MATLAB-2013b version- 8.1.0.604.
The evaluation of proposed iris recognition technique in benchmark iris image databases are carried out using the following metrics as suggested by below equations,

Accuracy: The Accuracy of the proposed method is the ratio of the total number of $T P$ and $T N$ to the total number of data.

$$
\text { Accuracy }=\frac{T N+T P}{(T N+T P+F N+F P)}
$$

Where, FP stands for false positive, TP stands for true positive, $T N$ stands for true negative, $F N$ stands for false negative

False Acceptance Rate (FAR): FAR is the probability rate at which numbers of Iris images are erroneously received as "non-match.

False Rejection Rate (FRR): FRR is the probability rate at which the numbers of iris images are erroneously received as Match.

\subsection{Effectiveness of the segmentation performance}

The accuracy of iris recognition highly depends on the accuracy of segmenting the iris and pupil or region of interest. In this context, N. P. Bodorin [26] has developed an approach to iris segmentation.

Figure 2 illustrates the segmentation performance of the proposed against existing approach namely circular fuzzy segmentation (CFS) [26].

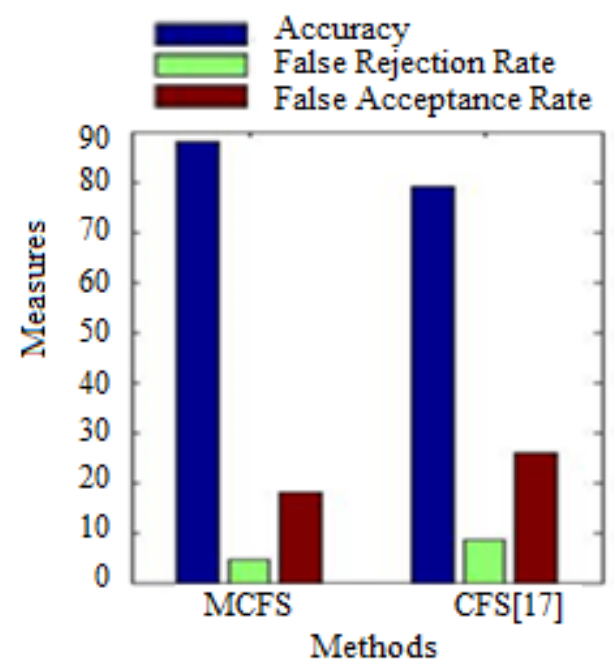

Figure.2 Segmentation performance of the proposed against existing system [26] 
Table 1. Visual segmentation results of proposed against existing approach [26]

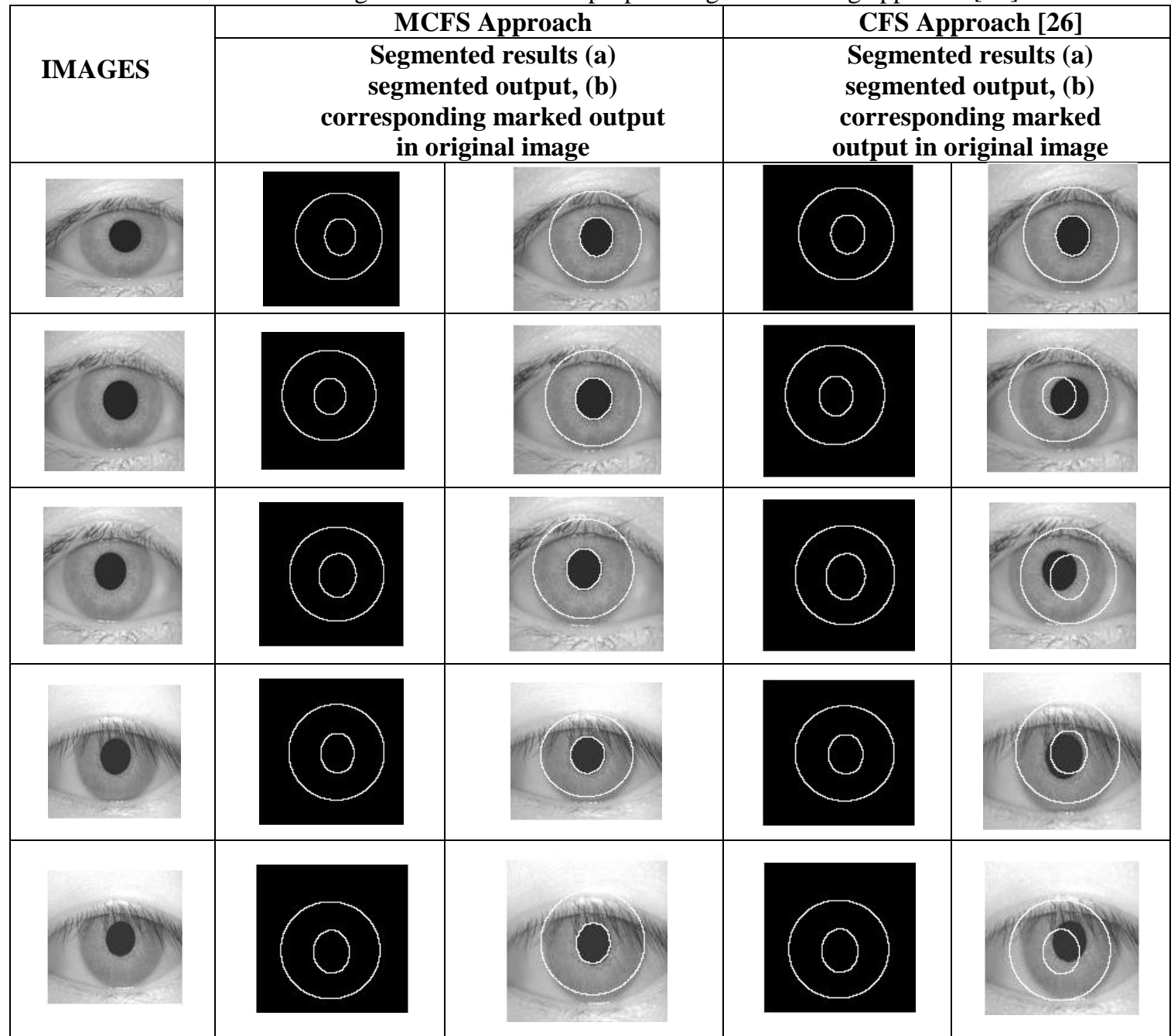

The figure 2 indicate that the proposed segmentation method of MCFS performs better accuracy than the CFS [26] method. From the figure 2 , our MCFS method achieves better accuracy value of $88 \%$, which is higher than the CFS algorithm (78\%). Overall, figure 2 reports a fact: some of the irises could not be segmented accurately using CFS algorithm. However, our proposed method of MCFS was segmented maximum number of iris images than the CFS algorithm. In addition, table 1 shows the visual representation of segmentation results.

\subsection{Effectiveness of the recognition performance}

The methods proposed by PBIM approach [27], SURF approach [28] are the best known among existing systems for iris recognition. In [27], phase based iris recognition is proposed using Fourier Phase Code (FPC) for representing iris information. A major problem of their approach is that the 2D FPC does not contain amplitude spectrum and the actual iris image cannot be reconstructed from the 2D FPC. In [28], the authors have developed an iris recognition system. However, their system is developed to localize the inner pupil boundary accurately for most samples from the CASIAV3 database but fails to localize the outer iris boundary. However, errors due to localization increase for the noisy and low resolution and UBIRIS databases. The proposed method is performed on three benchmark datasets, CASIA, MMU and UBIRIS. Hybridization of modified k-means and fuzzy logic classifier is well suited to improve the recognition process. The performance of the proposed recognition method is analysed by accuracy, false acceptance rate (FAR) and false rejection rate (FRR) and presented in figures 3, 4 and 5 .

As seen in the figures, the proposed method of MCFC-LCE had the highest accuracy, FAR and FRR rate for all iris images compared to the existing methods. 


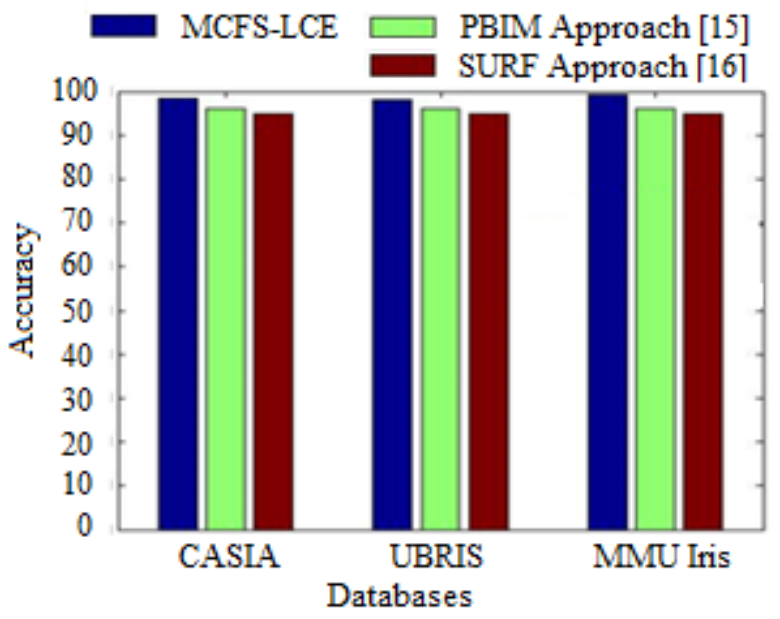

Figure.3 Recognition performance of the proposed against existing systems [27] and [28] based Accuracy measure

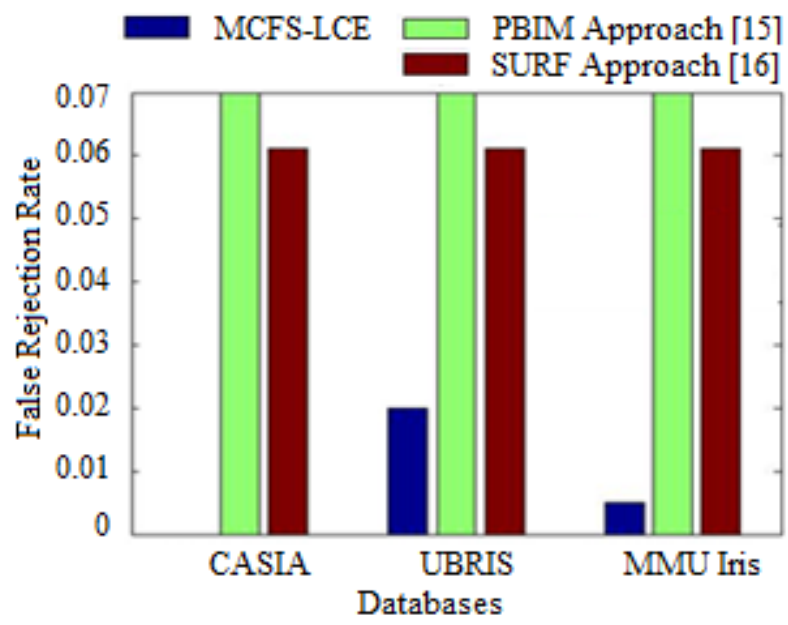

Figure.4 Recognition performance of the proposed against existing systems [27] and [28] based FRR

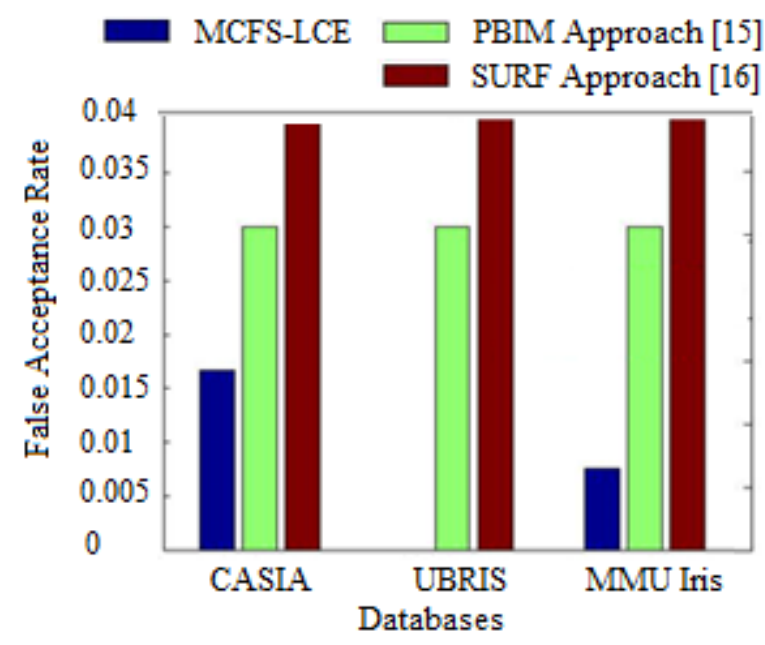

Figure.5 Recognition performance of the proposed against existing systems [27] and [28] based FAR measure
For example (for UBIRIS database), the proposed method (MCFC-LCE) gave $99.14 \%$ of accuracy and 0.0076 of FAR and 0.0051 of FRR, the PBIM approach [15] which gave $87 \%$ of accuracy and $45 \%$ of FAR and $7 \%$ of FRR, followed by the SURF approach [16] which gave $94 \%$ of accuracy and $39 \%$ of FAR and $6 \%$ of FRR. Overall, the proposed method of MCFC gives the better recognition results than the existing algorithms.

\section{Conclusion}

In this study, an iris segmentation and recognition method, called modified circular fuzzy segmentor and local circular encoder (MCFS-LCE), was proposed. At first, we utilize the modified circular fuzzy segmentor (MCFS) model to segment the pupil and iris inner boundary. After that, a binary encoder based feature extraction scheme named as LCE is proposed to extract the significant features to do the iris recognition process. Once feature extraction scheme is done by the LCE operator, the iris recognition is done through fuzzy logic classifier. Our tests on a more challenging iris image database set, CASI, MMU, UBIRIS, confirmed that our segmentation method is robust and results in performance gains. An experimental result indicates that, the proposed method of MCFS+LCE is outperformed than the existing methods. Our future work includes how to improve the robustness to segmentation error with different real-time and benchmark datasets.

\section{References}

[1] E. Lupu and P. G. Pop, "Multimodal Biometric Systems Overview", Acta Technica Napocensis, Electronics and Telecommunications, Vol. 49, No. 3, 2008.

[2] K. Jain, R. Bolle and S. Pankanti, eds., "Biometrics: Personal Identification in Networked Society", Kluwer Academic Publishers, Vol. 479, 1999.

[3] D. Zhang, "Biometrics Technologies and Applications", Proc. of International Conference on Image and Graphics, Tianjing, China, pp. 249-254, 2000.

[4] G. Lawton, "Biometrics: A New Era in Security", IEEE Computer, pp. 16-18, Aug. 1998.

[5] H. Zhuang, T. Theerawong, X. Guan and S. Morgera, "A Method for Creating 3D Face from a 2D Face Image", Florida Conference on Recent Advances in Robotics (FCRAR 2006), Miami, Florida, May, 2006. 
[6] V. S. Meenakshi and G. Padmavathi, "Securing Iris Templates using Combined User and Soft Biometric based Password Hardened Fuzzy Vault", (IJCSIS) International Journal of Computer Science and Information Security, Vol. 7, No. 2, 2010.

[7] M. Baca and K. Rabuzin, "Biometrics in Network Security", The Proceedings of the XXVIII International Convention MIPRO 2005, Rijeka, pp. 205-210, 2005.

[8] K. Jain, A. Ross and S. Prabhakar, "An introduction to biometric recognition", IEEE Trans. on Circuits and Systems for Video Technology, Vol. 14, No. 1, pp. 4-20, 2004.

[9] G. Chellin, J. Chandran and R. S. Rajesh, "Performance Analysis of Multimodal Biometric System Authentication", IJCSNS International Journal of Computer Science and Network Security, Vol. 9, No. 3, 2009.

[10] P. Manikandan and M. Sundararajan, "Discrete Wavelet Features Extraction for Iris Recognition Based Bio Metric Security", International Journal of Electronic Engineering Research, Vol. 2, No. 2, pp. 237-241, 2010.

[11] C. Y. Yam, M. S. Nixon and J. N. Carter, "Gait Recognition by Walking and Running: A Model-Based Approach", ACCV 2002: The 5th Asian Conference on Computer Vision, pp. 1-6, 2002.

[12] L. Ma, T. Tan, Y. Wang and D. Zhang, "Personal Recognition Based on Iris Texture Analysis", IEEE Transactions on Pattern Analysis and Machine Intelligence, Vol. 25, No. 12, pp. 1519-1533, 2003.

[13] A. M. Sarhan, "Iris Recognition Using Discrete Cosine Transform and Artificial Neural Networks", Journal of Computer Science, Vol. 5, No. 5, pp. 369-373, 2009.

[14] S. Dey and D. Samanta, "Improved Feature Processing for Iris Biometric Authentication System", International Journal of Computer Systems Science and Engineering (IJCSSE), World Academy of Science, Vol. 4, No. 2, pp. 127-134, 2008.

[15] S. Dey and D. Samanta, "A Novel Approach To Iris Localization For Iris Biometric Processing", International Journal of Biological and Life Sciences, Vol. 3, No. 3, pp. 180-191, 2007.

[16] W. Boles and B. Boashash, "A human identification technique using images of the iris and wavelet transform", IEEE Transaction of Signal Processing, Vol. 46, No. 4, pp. 11851188, 1998.
[17] S. Lim, K. Lee, O. Byeon and T. Kim, "Efficient iris recognition through improvement of feature vector and classifier", Journal of Electrical Technical Research Institute, Vol. 23, No. 2, pp. 61-70, 2001.

[18] S. P. Narote, A. S. Narote and L. M. Waghmare, "Iris Based Recognition System using Wavelet Transform", International Journal of Computer Science and Network Security, Vol. 9, No. 11, pp. 101, November 2009.

[19] N. P. Bodorin, "Circular Fast Fuzzy Iris Segmentation \& Fast k-Means Quantization Demo Programs", June 2009,

[20] N. P. Bodorin, "Fast k-Means Image Quantization algorithm and its application to iris segmentation", Scientific Bulletin, University of Pitesti, No. 14, 2008.

[21] J. Daugman, "How Iris Recognition Works", In Proceedings of International Conference on Image Processing, Vol.1, pp. I-33- I-36, 2002.

[22] S. Chiu, "Fuzzy model identification based on cluster estimation," Journal of Intelligent \& Fuzzy Systems, Vol. 2, No. 3, pp. 267-278, 1994.

[23] H. Proença and L. A. Alexandre, "Ubiris: a noisy iris image database", In International Conference on Image Analysis and Processing, Vol. 3617, pp. 970-977, 2005.

[24] J. Horst, "Iris Recognition: A General Overview", Journal of Student Research, pp. 19-23, 2006.

[25] S. Uma Maheswari, P. Anbalagan and T. Priya, "Efficient Iris Recognition through Improvement in Iris Segmentation Algorithm", International Journal on Graphics, Vision and Image Processing, Vol. 8, No. 2, pp. 29-35, 2008.

[26] N. P. Bodorin, "Circular Fast Fuzzy Iris Segmentation", Department of Mathematics and Computer Science, Spiru Haret University, 2009.

[27] K. Miyazawa, K. Ito, T. Aoki, K. Kobayashi and H. Nakajima, "An Effective Approach for Iris Recognition Using Phase-Based Image Matching”, IEEE transactions on pattern analysis and machine intelligence, Vol. 30, No. 10, pp. 1741-1756, 2008.

[28] H. Mehrotra, P. K. Sa and B. Majhi, "Fast segmentation and adaptive SURF descriptor for iris recognition", Mathematical and Computer Modelling, Vol. 58, No. 1, pp. 132-146, 2013. 